\title{
CIENCIA, CONOCIMIENTO Y REALIDAD SOCIAL
}

\author{
Science, Knowledge and Social Reality
}

Marcelo Chinche Calizaya

Universidad Mayor de San Simón, Bolivia

\section{KEY WORDS}

Legitimation

Transdisciplinarity

Decolonization

Science

Knowledge

Fragmentation

Specialization

\section{ABSTRACT}

The present work aims to reflect on the frequent reorganization of knowledge, with marked tendencies towards higher levels of disciplinary specialization - without ruling out at any time superspecialization based on the division and subdivision of traditional areas of knowledge-, as a logical consequence of work and the deepening of scientific research, recognizing and valuing the fundamental role it plays in the explanation of a myriad of complex problems, a product of the scientific revolution experienced with greater notoriety since the end of the 20th century and the beginning of the 21st century. In fact, the investigative function has been widely favored as a result of the definitive installation of science and knowledge as structural components that qualify the development of the productive forces of contemporary societies.

\section{PALABRAS CLAVE}

Legitimación

Transdisciplinariedad

Decolonización

Ciencia

Conocimiento

Ideología

Saber

Valoración

Fragmentación

Especialización

\section{RESUMEN}

El presente trabajo pretende reflexionar acerca de la frecuente reorganización del conocimiento, con marcadas tendencias hacia mayores cotas de especialización disciplinar -sin descartar en ningún momento la superespecialización a partir de la división y subdivisión de áreas tradicionales del conocimiento-, como consecuencia lógica del trabajo y la profundización de la investigación científica, reconociendo y valorando el papel fundamental que cumple en la explicación de un sinfín de complejos problemas, producto de la revolución científica experimentada con mayor notoriedad desde fines del siglo XX e inicios del siglo XXI. De hecho, la función investigativa se ha visto ampliamente favorecida a consecuencia de la instalación definitiva de la ciencia y el conocimiento como componentes estructurales que cualifican el desarrollo de las fuerzas productivas de las sociedades contemporáneas.

Recibido: 05/02/2021

Aceptado: 09/02/2021

\section{GLOBAL KNOWLEDGE




\section{Abordaje del problema central}

$\mathrm{E}$ l inicio del nuevo siglo trajo consigo una marcada tendencia hacia la reorganización del conocimiento, que aspiran a consolidar mayores cotas de especialización por un lado $y$, por otro, a propensiones de cara hacia una aparente intencionalidad de unificación del saber (conocimiento). Ambas tendencias constituyen los nuevos polos entre los que va desarrollándose a manera de péndulo, la construcción y difusión del conocimiento.

La tendencia al logro de mayores de cotas de especialización, es consecuencia lógica del trabajo y la profundización de la investigación científica que llevan a cabo las personas en el marco de una especialidad concreta; que -las más de las veces-, ha conducido a una superespecialización, a partir de la división y subdivisión de algunas áreas tradicionales del conocimiento. No obstante, debe cuidarse que tal intencionalidad orientada hacia la especialización disciplinar, podría peligrosamente dar paso a hacer desaparecer el propio fenómeno (objeto de estudio), en razón a que se halla dividido en pequeños fragmentos o parcelas del saber científico, dejando de lado la importancia de comprender la globalidad y la totalidad del fenómeno en su integridad.

En un otro sentido, viene también operando otra dinámica impulsada por aquellas disciplinas que comparten objetos de estudio -parcelas de una misma temática o metodologías de investigación-, llegando a comunicarse y coordinarse hasta lograr la conformación de nuevos ámbitos interdisciplinares de conocimiento.

Al parecer, esta tendencia viene cobrando especial notoriedad en virtud a que nos hallamos situados frente a la ruptura progresiva de fronteras entre las disciplinas que está obligando a la adopción de nuevos y complejos modelos de análisis mucho más potentes; justificando con ello, la inviabilidad de seguir recurriendo a los modelos tradicionales de una única especialización disciplinar, para dar cuenta de los fenómenos del mundo social desde múltiples ópticas de abordaje integrales en el afán de evitar reduccionismos, fragmentaciones y hasta deformaciones en su abordaje y análisis.
Otro aspecto a contemplarse con especial interés es el referido a qué tipo de conocimientos son necesarios y pertinentes generar; cómo aprovecharlos y, sobre todo, cómo hacerlos en tanto éstos se hallen en correspondencia con la resolución de los grandes problemas actuales y en relación con el desarrollo económico, social y cultural de las sociedades históricamente determinados.

Ello implica sin duda, repensar el papel de las Instituciones de Educación Superior (IES) y particularmente las Universidades en la promoción de nuevos procesos de producción de conocimiento (saberes), a partir de una necesaria contextualización social del mismo; la adopción de nuevas propuestas alternativas de investigación, organización y apertura democrática del espacio universitario, como mecanismo indispensable $y$ esencial que contribuya a la efectiva reconexión con el contexto (espacio social, político, económico y cultural); la búsqueda de soluciones colectivas a los complejos problemas locales y nacionales, sin perder de vista el referente del escenario internacional -al cual nos hallamos interconectados-, que condiciona pero que también es condicionado por lo local.

\section{Argumentacion de las ideas centrales}

La frecuente reorganización del conocimiento, con tendencias marcadas hacia mayores cotas de especialización por un lado $\mathrm{y}$, por otro a propensiones de cara hacia una aparente intencionalidad de unificación del saber (conocimiento); representan los nuevos polos entre los que viene desarrollándose a manera de péndulo, la construcción y difusión del conocimiento. Dicha tendencia es consecuencia lógica del trabajo y la profundización de la investigación científica ${ }^{1}$ que llevan a cabo las

\footnotetext{
1 El reconocimiento y valoración creciente del papel fundamental que cumple la investigación en la explicación de un sinfín de complejos problemas, producto de la revolución científica experimentada con mayor notoriedad desde fines del siglo XX e inicios del Siglo XXI; sumados a la instalación definitiva de la ciencia y el conocimiento como componentes estructurales que cualifican el desarrollo de las fuerzas productivas de las sociedades, han dado lugar al reconocimiento y revalorización de la función investigativa,
} 
personas en el marco de una especialidad concreta; que -las más de las veces-, ha conducido a una superespecialización ${ }^{2}$, a partir de la división y subdivisión de algunas áreas tradicionales del conocimiento.

Sin embargo, no hay que olvidar que esta tendencia hacia la especialización disciplinar, peligrosamente tiende a hacer desaparecer el propio fenómeno (objeto de estudio), en razón a que se halla dividido en pequeños fragmentos o parcelas del saber científico, dejando de lado la importancia de comprender la globalidad y la totalidad del fenómeno en su integridad.

En un otro sentido, viene también operando otra dinámica impulsada por aquellas disciplinas que comparten objetos de estudio -parcelas de un mismo tema o metodologías de investigación-, llegando a comunicarse y coordinarse hasta lograr la conformación de nuevos ámbitos interdisciplinares de conocimiento.

Desde el punto de vista de la teoría del conocimiento, es decir, como doctrina, puede afirmarse que "todo conocimiento cierto viene de principios irrecusables, apriori, evidentes de lo que es la consecuencia necesaria, ya que los sentidos solos no pueden suministrar más que una visión confusa y provisional de la verdad" (Di Tella, 2008: 587).

Otra dinámica, ampliamente favorecida por el surgimiento del fenómeno de la superespecialización y los procesos de interdisciplinariedad del conocimiento, es el referido a la conformación de equipos de investigación con una perspectiva interdisciplinar, expresada en el concurso de

como pilar fundamental que posibilita el progreso y desarrollo sostenido de la ciencia y el conocimiento en las distintas áreas y disciplinas científicas, situándola en un lugar privilegiado; en razón a que ella constituye la herramienta esencial para desarrollar el conocimiento y por ser el mecanismo privilegiado a través del cual, es posible comunicar y transferir el conocimiento a diferentes campos disciplinares, contextos y regiones. Por ello, puede ser considerado el idioma universal de la ciencia que posibilita el desarrollo y progreso del conocimiento en todos los campos, el intercambio y transferencia de tecnología, así como el establecimiento de acuerdos y consensos en el trabajo multi, inter y transdisciplinar.

2 La superespecialización surge como resultado de la división y subdivisión de áreas del conocimiento y en la actualidad, viene cobrando mayores niveles de independencia y autonomía de parcelas o temáticas muy específicas de algunos campos de investigación considerados dominantes. varios campos de conocimiento e investigación a la hora de tratar, comprender y solucionar los complejos problemas de la realidad.

Lo cierto es que hoy, nos hallamos frente a una progresiva ruptura de fronteras entre las disciplinas que ha obligado a la adopción de nuevos y complejos modelos de análisis mucho más potentes que hacen inviable seguir recurriendo a los modelos tradicionales de una única especialización disciplinar, debido en gran manera, al reconocimiento de la complejidad del mundo y la cultura, que exigen desentrañar los problemas actuales desde múltiples ópticas de abordaje que revisten -desde ya-, especial interés y preocupación para la comunidad científica.

Sumado ello, resulta imprescindible el concurso de las áreas de conocimiento (mirada interdisciplinar), cuyas aspiraciones sean por un lado, evitar que los resultados se vean afectados por reduccionismos, fragmentaciones $\mathrm{y}$ hasta deformaciones en su abordaje y análisis y, por otro, incrementar las capacidades de descripción teórica y empírica de todo aquello que sucede en el objeto de análisis; en razón a que "juzgamos el conocimiento por aquello que puede mostrar (en lo teórico y empírico) y no por su adecuación respecto de algún patrón externo" (Follari, 2000: 104-105).

Sin embargo, cabe reconocer prontamente que esta tendencia no ha sido del todo positiva; pues, ha dado paso al surgimiento de nuevos cuestionamientos relativos a qué tipo de conocimientos son necesarios generar; cómo aprovecharlos y, sobre todo, cómo hacerlos en tanto éstos se hallen en correspondencia con la resolución de los grandes problemas actuales y en relación con el desarrollo económico y social de un contexto sociocultural determinado históricamente.

Cuestionamientos tales que se hallan impulsados principalmente por aquellas sociedades cuyo desarrollo económico y social, se sustenta en el conocimiento científico y tecnológico -con pretensiones hegemónicas-, que a decir de ellos, constituye el trabajo intelectual altamente calificado como base fundante para la generación de riqueza y el desarrollo de la ciencia; que bien podría ser entendida como la progresiva mercantilización 
del conocimiento y sobre todo, la instalación de un imperialismo cultural hegemónico, que "ante todo, es asunto de la mecánica de fuerzas de un sistema de poder, de un engranaje de relaciones desiguales de donde resulta la hegemonía de una visión del mundo" (Mattelart. 2006:80).

Pero este imperialismo cultural, no se reduce única y exclusivamente a las manifestaciones de las relaciones de fuerza en los medios de comunicación y de la cultura de masas, sino que se expresa con mayor fuerza en los "modelos de institucionalización de las tecnologías de comunicación, los modos de organización espacial, los paradigmas científicos, los esquemas de consumo y de aspiraciones, los modos de gestión de la empresa, los sistemas de alianzas militares" (Mattelart. 2006:81), entre otros aspectos.

Estos nuevos modos de concebir el conocimiento científico y tecnológico, han llevado a las Instituciones de Educación Superior (IES) y particularmente, a las Universidades consideradas centros de desarrollo y producción de ciencia y conocimiento, a repensar su accionar en torno a promover la profundización de aquello que se ha venido a llamar la mercantilización del conocimiento o en su defecto, pensar en la posibilidad de transformación de los procesos de conocimiento (saberes), a partir de una necesaria contextualización social del mismo.

Adscribirse a esta segunda opción, exige la adopción de nuevas alternativas de investigación, organización y apertura democrática del espacio universitario, como mecanismo indispensable $y$ esencial que contribuya a la efectiva reconexión con el contexto (espacio social político, económico y cultural); la búsqueda de soluciones colectivas a los complejos problemas locales, regionales y nacionales, sin perder de vista el referente del escenario internacional -al cual nos hallamos interconectados-, que condiciona pero que también es condicionado por lo local.

Desde el momento en que el sistema capitalista se ha tornado planetario, ha dado paso a que la Universidad -por inercia-, vaya plegándose progresivamente a los imperativos del mercado global -a consecuencia de la irrupción de la globalización-; pero conduciéndola en contrapartida, a una situación de crisis de legitimación, que es consecuencia directa de la pérdida progresiva del lugar y/o espacio privilegiado de referencia que ostentaba en la producción de ciencia y conocimiento. Tal apreciación tiende a ser aún más evidente, en razón a que actualmente, el saber hegemónico y la producción de conocimientos son desarrollados por grupos, equipos y empresas corporativas transnacionales y multinacionales especializadas, totalmente ajenas al espacio universitario -las más de las veces-, y que realizan inversiones millonarias en investigación y desarrollo de ciencia y tecnología.

Ello es ampliamente comprensible, dado que "la actividad científica se engendra en la relación entre las disposiciones reguladas de un habitus científico que en parte es producto de la incorporación de la necesidad inmanente del campo científico y las imposiciones estructurales ejercidas por este campo en un momento dado del tiempo" (Bourdieu. 1997: 89). A esta situación, también ha contribuido negativamente el Estado que -en el caso boliviano-, "descapitaliza la universidad pública porque para este proceso de desarrollo capitalista la universidad pública ya no es necesaria; está suspendida en el aire, sin un bloque histórico que la pueda sustentar" (De Sousa Santos, 2008: 90).

Tales factores han dado paso a la lógica consecuencia de que el conocimiento hegemónico de referencia, ya no se produce en la Universidad, sino que es incentivado y producido por un mercado que -a la forma de corporaciones, equipos y laboratorios especializados-, la determina, orienta y fiscaliza para generar determinados efectos de poder $y$ control; además de promover una especie de viraje al interior de la universidad, expresado en la priorización de inversiones para el rubro de la investigación como mecanismo y alternativa eficaz que viabilice la producción de conocimientos pertinentes y útiles para el mundo globalizado y la sociedad del conocimiento. Desnudando con ello, la intencionalidad y obligatoriedad de las Instituciones de Educación Superior y de manera especial la Universidad de adaptarse a las demandas complejas del mercado y la elaboración de productos que se ajusten eficazmente a las necesidades de los consumidores. 
Con base en tales argumentos, el concepto de conocimiento pertinente $y$ útil resulta ser demasiado determinante en términos epistémicos; pues da pie a una especie de ambivalencia e indecisión práctica con relación a aquello que "es" o "debe" considerarse como metas del conocimiento científico; situando nuevamente a la Universidad en la encrucijada de construir conocimiento científico y/o la formación de profesionales altamente calificados y competitivos que dispongan de un conocimiento pertinente y útil al contexto actual.

Esta nueva dinámica explica en algún modo, el debilitamiento del espacio universitario a consecuencia de las modificaciones del desarrollo y producción del conocimiento que impulsa la transición del "conocimiento universitario convencional hacia el conocimiento pluriuniversitario, transdisciplinar, contextualizado, interactivo, producido y consumido con base en las nuevas tecnologías de la comunicación e información que alteraron por un lado, las relaciones entre conocimiento $e$ información y por otro lado, entre formación y ciudadanía" (De Sousa Santos, 2007: 58).

$\mathrm{Al}$ parecer, la necesidad de potenciar la investigación para la producción de conocimiento pertinentes y útiles y la superación de la ambivalencia e indecisión práctica de aquello que "es" o "debe" considerarse como metas del conocimiento, constituyen las nuevas interrogantes que deben ser resueltas con prontitud por la Universidad, en tanto generadora de conocimiento y productora de la ciencia.

Un otro desafío estructural que debe afrontar la Universidad del Siglo XXI -después de constatar la pérdida de hegemonía-, se halla orientada a la cuestión de reconquistar la legitimidad y el reconocimiento de su aporte efectivo al desarrollo de ciencia y conocimiento; el fomento a las políticas de emprendimiento e iniciativas que viabilicen el posicionamiento $\mathrm{y}$ consolidación de un conocimiento pluriuniversitario; además de ratificar su compromiso, responsabilidad social y solidaria con la sociedad.

Lograr este ideal supremo, plantea a la Universidad como condición sine qua non, la extensión de puentes teórico-conceptuales no tanto en un sentido interno de afianzamiento, sino más bien, en un sentido de extensión desde afuera hacia dentro del espacio universitario, que posibilite la "promoción de diálogos entre el saber científico y humanístico que la universidad produce y los saberes legos, populares, tradicionales, urbanos, campesinos, provincianos, de culturas no occidentales... que circulan en la sociedad" (De Sousa Santos, 2007: 67).

No obstante, esta promoción y vinculación de diálogos entre los saberes populares y el saber científico ${ }^{3}$, exige previamente decolonizar la Universidad de la dominación cultural de los saberes -que refleja un imperialismo cultural hegemónico-, que ha socavado y penetrado intensa y sistemáticamente en nuestras mentalidades, pensamientos y subjetividades, quebrantando la profunda y rica densidad histórica y cultural que poseen nuestros pueblos. Situación que es ampliamente comprensible en razón a que la "Universidad, al especializarse en el conocimiento científico y al considerarlo la única forma de conocimiento válido, contribuyó activamente a la descalificación e inclusive a la destrucción de mucho conocimiento no científico y con eso, contribuyó a la marginalización de los grupos sociales que solamente disponían de esas formas de conocimiento" (De Sousa Santos, 2007: 67).

El esfuerzo de afrontar la decolonización conlleva "revertir la colonialidad del poder del saber y del ser... implica la reconstitución y el restablecimiento de los saberes, los conocimientos, las ciencias y las culturas históricamente subalternizadas" (Saavedra, 2011: 136-137), dando lugar a la restitución, reintegración y revalorización de aquellos saberes que histórica y deliberadamente han sido sometidos y/o puestos en segundo plano de interés expectaticio para preservar el statu quo del imperialismo científico cultural hegemónico imperante.

Afrontar responsablemente estos desafíos, pasa por desarrollar en el espacio universitario,

\footnotetext{
${ }^{3}$ En la actualidad, es posible apreciar ciertos acuerdos y consensos a la hora de considerar que la ciencia es una forma -más- de saber o de conocimiento; pues es posible visualizar y reconocer que existen muchos conocimientos y saberes que no son científicos (saberes populares), y que no pretenden serlo. Lo cual implica aceptar esa rica pluralidad en el abordaje de los complejos fenómenos y problemas sociales, rechazando tácitamente aquel supuesto de que sólo la ciencia es un saber, y los otros -saberes popularesson sólo creencias.
} 
prácticas no convencionales que den paso a la generación de encuentros horizontales que posibiliten la apertura del diálogo de saberes prácticos considerados no-científicos y aquellos considerados científicos; de tal forma que ambas puedan enriquecerse mutuamente $y$, producto de ese encuentro, se vayan conformando comunidades epistémicas donde se desarrolle ciencia y conocimiento útil y contextualizado; que permita intervenir socialmente desde el conocimiento, además de "ejercer una adecuada crítica metodológica y una permanente vigilancia epistémica sobre los actos teóricos fundacionales de las imágenes científicas que se construyen en el mundo social" (Scribano. 2009:18).

De hecho, esto es posible en la actualidad, gracias a que la epistemología contemporánea ha logrado superar el prejuicio positivista de que la ciencia era el único saber; asumiendo que "hoy lo epistemológico se va constituyendo como reflexión sistemática acerca de las ciencias, auxiliada por éstas (en cuanto epistemología general); y como autoconciencia conceptual de las ciencias mismas" (Follari, 2000: 38).

Nótese que utilizamos el concepto "saber" en sustitución de "conocimiento", puesto que éste último se halla plenamente identificado con las formas más sistemáticas y rigurosas del saber científico y el saber filosófico.

Ahora bien, hablar de ciencia bajo los nuevos discursos y estatutos de la epistemología contemporánea, parte de supuestos un tanto alejados del convencionalismo clásico con los que antaño $0^{4}$ operaba principalmente, en razón a que la filosofía sobre las ciencias ya no puede realizarse prescindiendo de ésta última. Vale decir que, en la actualidad, la epistemología "ya no se plantea como exclusivamente filosófica, sino que requiere lo científico como elemento constituyente de sí" (Follari, 2000: 38).

Esto es ampliamente comprensible por su preocupación por el conocimiento sobre la investigación -en el fondo, la ciencia-, lo que hace y busca, considerando en este proceso los determinantes sociales -en este caso, los

\footnotetext{
$4 \mathrm{Al}$ parecer, la epistemología progresivamente ha ido perdiendo aquella omnipotencia construida tras sí misma, desde el cual tenía la potestad de emitir juicios valorativos acerca de toda actividad científica, la pretensión normativa de regentar y orientar la producción y reproducción concreta de la ciencia.
}

instrumentos sociológicos y hasta psicológicos que permitan apreciar la naturaleza, la particularidad y especificidad de los objetos y fenómenos que interesan al mundo académico-, que la inundan de principio a fin; los posicionamientos teórico-filosóficos que asumen los intelectuales en el debate científico; además de aquellos espacios y lugares de adscripción que ocupan dentro de la comunidad científica.

Por ello, adentrarnos en la comprensión de la ciencia, conlleva ir asumiendo algunos reparos de orden no sólo conceptual sino principalmente de orden epistemológico, en tanto que no se debe olvidar que la misma se desarrolla en un determinado contexto sociohistórico, pero que también se halla cargada de orientaciones de orden ideológico y valorativo que la condicionan y que van más allá del mero planteamiento que representa la posibilidad viable y válida de producción y construcción de conocimiento, como resultado de la negación y superación progresiva de incertidumbres.

Asimismo, conviene dejar establecido que la ciencia, bajo ninguna circunstancia puede pretender de forma cerrada y definitiva, determinar cómo son los hechos o fenómenos que estudia, sino simplemente representa una posibilidad -entre otras formas y maneras de abordaje de los hechos y/o fenómenos-, una intencionalidad metodológica, lógica, coherente de explicación del comportamiento ideal de algunas normas y leyes que operan en el objeto específico de análisis o de estudio. De hecho, la ciencia puede ser advertida "en lo que tiene de objetivación, más que de objetividad, es el fruto de una cierta forma de poner los objetos en perspectiva, de captar sus aspectos legaliformes y repetibles, de modo de hacer desaparecer de la percepción aquello que queda fuera de dicho campo de estipulación pura" (Follari, 2000: 11).

Es más, la ciencia lejos de intentar explicar cómo son los hechos (certidumbre total), simplemente intenta describir aquellas mutaciones, crisis y evoluciones que resultan ser más importantes y trascendentales que -a nuestro juicio, son más significativos $y$ relevantes-, sólo presentar las regularidades y/o normalidades de los hechos. Por otro lado, tampoco debe suponerse que la ciencia surge de la mera observación; pues ella parte 
necesariamente de supuestos previos que son sujetos de contrastación empírica a través de la experiencia, guiados por un cuerpo teóricometodológico riguroso no convencional, que se encuentra en constante modificación y adaptación a la dinámica del contexto actual.

Desde tales orientaciones, es posible plantear que la ciencia tiene como objeto, "el conjunto de lo que existe empíricamente -a esto puede llamársele universo- y lo que trata con tanta precisión...", pero también la ciencia moderna "en tanto empírica, no es tan sólo experimental, es instrumental" (Milner, 1996: 46-47).

A partir del momento que la ciencia toma lo empírico que históricamente ha representado el tratamiento material -esto por los instrumentos producidos por la técnica-, se halla en la obligatoriedad de proporcionar sus instrumentos, que permiten legitimar que ella sea considerada como ciencia precisa. 0 lo que es lo mismo, la ciencia sólo es precisa si los instrumentos producidos por la técnica se lo permiten materialmente.

El universo de la "ciencia moderna es, al mismo tiempo y en el mismo movimiento, un universo de la precisión y un universo de la técnica" (Milner, 1996: 48), que se halla configurado por la unión recíproca de la ciencia y la técnica que bien puede expresarse como una ciencia fundamental aplicada o como una técnica teórica o práctica.

Cabe enfatizar que la ciencia en la actualidad, debe enfrentarse a "un universo fragmentado y a una pérdida de certidumbre, a un nuevo examen del lugar del azar y del desorden, así como a una toma en consideración de las evoluciones, mutaciones, crisis y trastornos en lugar de los estados estables y permanentes" (Pourtois; Desmet, 1992: 18); que a su vez, implica la realización de profundas modificaciones a las metodologías y aquellas lógicas de comprensión de la realidad "porque se trata de considerar el cambio y no ya los estados estables" (Pourtois; Desmet, 1992: 18), que caracterizan el devenir socio histórico en el que se inserta la producción de ciencia y conocimiento .

Por ello, cualquier análisis crítico-reflexivo referido a la constitución de las Ciencias, debe considerar necesariamente su origen y desarrollo en lo social; pues no debe olvidarse que son construcciones sociales resultantes del hacer y quehacer social (hechos sociales), ligados a determinadas condiciones de poder bajo las cuales se producen, difunden y aplican (ideologías).

Con lo que se ratifica que el saber científico es, por esencia, un saber cargado de ideología producida en aquellos hechos sociales "construidos socialmente, $y$-donde- todo agente social, como el científico, construye de mejor o peor manera, y tiende a imponer, con mayor o menor fuerza, su singular visión de la realidad, su punto de vista" (Bourdieu, 2003: 153).

A la par de ello, podemos mencionar también que la ciencia "no está en simple continuidad con las ideas de quienes la hacen, y la enorme distancia entre un trabajo de investigación y un manifiesto ideológico" (Follari, 2000: 11); en virtud a que el desarrollo de la ciencia no se encuentra ajena a las intencionalidades $\mathrm{u}$ orientaciones (intereses, motivaciones e inclinaciones) propias de los investigadores en tanto sujetos sociales, que formulan posibles explicaciones que intentan pensar la realidad social, definiendo y construyendo para ello, marcos conceptuales corpus teórico- e instrumentos, producto del trabajo científico que buscan aproximarse a la comprensión de la realidad.

Esta situación explica la dinámica interna que opera en las comunidades científicas que se hallan en permanente redefinición de sus marcos conceptuales y cuya expectativa no es otra, que la construcción de nuevas posibilidades de comprensión del objeto de estudio; asumiendo que resulta imposible atraparlos enteramente, ya que siempre subsiste una distancia visibilizada o invisibilizada entre los resultados de la investigación y la aspiración o expectativa a lograr la totalidad y completitud de la comprensión del objeto de estudio. Así los objetos de conocimiento construidos por los científicos tienen a la realidad como su límite.

Más aún en el caso de las Ciencias Sociales, lo "real es absolutamente exterior e independiente del conocimiento, pero es a su vez una construcción social, un producto de las luchas anteriores que, por esas mismas razones, sigue siendo un objetivo de luchas actuales" (Bourdieu, 2003: 153). Aspectos tales, que orientan en algún modo, la constitución de la teoría científica que debe asumir entre otros requisitos, condiciones 
de rigurosidad, coherencia y contenido empírico que se obtienen a través de la interpretación y valoración de lo "social"; pues lo "característico de una teoría científica es ser siempre eventualmente comprobable y refutable y, por lo demás, toda historia de la ciencia nos muestra que las teorías son biodegradables" (Morin, 1995: 41).

Así como la ciencia no se encuentra despojada de la ideología, o lo que es lo mismo, el saber científico es un saber cargado de ideología; resulta también acertado señalar que no existe conocimiento específicamente social ni conocimiento general que se limite a reflejar de forma pasiva el mundo exterior y el sujeto, desde una neutralidad supuesta libre de valores. Ejemplo de ello es lo que sucede con el sujeto; pues la ciencia moderna, determina simultáneamente un modo de constitución del sujeto de estudio, el cual no es vacío, ya que posee una historia ${ }^{5}$, emociones, afectos e inclinaciones; lo cual hace suponer, por un lado, que la noción de ciencia constituye el objeto de una determinada teoría y por otro, asumir que esta teoría -una vez admitida-, tenga la capacidad de vincular cierta constitución del sujeto histórico.

Este aspecto resulta ser más notorio aún en el caso de las Ciencias Sociales, donde observar el mundo exterior desde una -supuestaneutralidad valorativa es poco menos que imposible. La valoración es "parte de la ciencia, y que no podemos imaginar ningún conocimiento social desinteresado, lo cual no está en contradicción con la búsqueda de racionalidad en el pensamiento" (Aisenberg; Alderoqui, 2007: 30); por lo que no resulta erróneo indicar, que tanto el conocimiento como los valores lejos de oponerse el uno hacia el otro, más bien se constituyen y autoconstituyen recíprocamente.

La ciencia al ser "un saber público, no es individual $y$, si bien los valores no pueden estar sujetos a verificación, el desarrollo teórico que los sustenta sí puede, y debe ser validado. Porque lo que está en juego no son los valores individuales, sino que se trata de valores socialmente relevantes. Y esta relevancia puede, en efecto, ser

\footnotetext{
5 En esa lógica la historia aparece como una factibilidad de múltiples posibilidades, como una forma de percepción circunstancial asumida por una conciencia determinada que bajo ninguna circunstancia tiene vida propia, independiente y autónoma fuera del sujeto.
}

demostrada" (Aisenberg; Alderoqui, 2007: 30). En tal sentido, puede afirmarse que tanto el conocimiento como los valores, no se oponen o reducen recíprocamente el uno al otro, al contrario, se constituyen y autoconstituyen conjuntamente.

De igual forma, la ciencia debe tener la capacidad de auto representarse a sí misma respecto al lugar que ocupa en relación a lo social; es decir, que sea capaz de pensarse a sí misma como socialmente producida.

Si la ciencia "tiene algún significado para la sociedad, es aquel de servir a modificar sus puntos de vista, a enriquecer sus posibilidades argumentativas, a incrementar su caudal informativo" (Follari.2000:40), que necesariamente parte de la existencia de posibilidades y oportunidades de socialización de la ciencia de reabsorberla socialmente, cuya finalidad no es otra, que la negación dialéctica de su especificidad por aquellos que no se encuentran incluidos en dicha especificidad práctica o discursiva (proceso dialéctico) ${ }^{6}$.

Este proceso dialéctico comporta dos dimensiones fundamentales que son la "unidad del ser y del pensar y la permanente evolución del espíritu y de la naturaleza a través de oposiciones sistemáticas que se superan unas a otras" (Di Tella, 2008: 189).

Un aspecto que diferencia notablemente el conocimiento social, es que éste se halla dirigido a comprender la trama significativa de aquellos espacios, lugares y/o prácticas sociales, donde se desarrollan un cúmulo de relaciones e interacciones dinámicas que dificultan notablemente el establecimiento de regularidades, normalidades y posibilidades de predicción estables.

Además, esta trama de relaciones e interacciones que tiene lugar en el conocimiento social, presentan cierta autonomía e intencionalidades relativas, dadas las estrategias para procesar la información recabada y que son elaborados en determinados contextos de significación, siendo precisamente éstas

${ }^{6}$ El proceso dialéctico queda plasmado en la formulación de un concepto o postura determinada (tesis), la cual inmediatamente se enfrenta a su opuesto (antítesis) y como resultado de esa confrontación y/o conflicto se arriba a una posibilidad superación y consecuente constitución de la verdad (síntesis). 
prácticas sociales las que diferencian y particularizan notablemente el conocimiento de la realidad social.

Dada esta dinámica interna y forma de concebir el conocimiento en las Ciencias Sociales, resultan estériles aquellos esfuerzos teóricometodológicos por emular de forma repetitiva y convencional, aquello que se hace en las Ciencias Naturales y aplicarlas analógicamente en la comprensión del mundo social en el caso de las Ciencias Sociales; generando consecuentemente, ansiedades $y$ tensiones irrelevantes e improductivas, al no poder ser tan exactos en comparación con las disciplinas de las Ciencias Naturales.

La realidad social es compleja, contradictoria, difícil de aprehender y que exige a los cientistas sociales, construir explicaciones a partir de las distintas concepciones que tienen acerca de la historia y de su sentido, a la luz de una teoría y un marco conceptual referencial que permita organizar y jerarquizar la información obtenida, en la intención de hallar generalizaciones de referencia necesarias para alcanzar la comprensión de aquellos objetos sociales que desde ya- poseen características peculiares que tienen que ver con el modo de abordarlos; toda vez que los sujetos que conocen la realidad, son sujetos sociales que intentan conocer a un objeto que a ellos mismos los constituye.

El cientista social debe tomar en cuenta estos aspectos que caracterizan a la realidad social; asumiendo como dato referencial de su labor investigativa, el lugar social que él mismo ocupa en tanto sujeto social instalado en un tiempo y espacio histórico determinado; la posibilidad de estudiarse a sí mismo y como objeto sociológico, para así poder conocer sus propias determinaciones, así como los posibles sesgos existentes con la finalidad de reducirlos y minimizarlos progresivamente. El hecho de estar consciente del lugar social que ocupa dentro el fenómeno objeto de estudio, conduce a su vez, el desafío de ir más allá de la realidad observada. Es decir, no sólo debe plantearse soluciones, sino también intentar formular críticas reflexivas acerca del problema social; toda vez que tanto la crítica como la solución son irreductibles e inseparables entre sí, al extremo de considerar que la formulación de cualquier crítica acerca del problema social, necesariamente debe implicar posibilidades $\mathrm{y} / \mathrm{o}$ alternativas de solución al mismo.

Para finalizar este análisis, debemos mencionar que el conocimiento, jamás revela sus propias fronteras como para que los cientistas sociales presuman haber logrado alcanzarla en su totalidad. Al contrario, creemos pertinente contribuir al desarrollo y constitución de un acto de apertura ${ }^{7}$ en el pensamiento científico, el surgimiento y consolidación de una conciencia crítica-reflexiva, en el sentido de establecer que nos encontramos cada vez más cerca de una incomprensión de la totalidad de la completitud; de que jamás llegaremos a poseer una comprensión cabal del mismo, y que sólo nos encontramos cada vez más cerca de parcialidades segmentadas de la verdad; generando con ello, rupturas definitivas con las clásicas afirmaciones construidas como proposiciones verdaderas, por el sólo hecho de provenir de evidencias y demostraciones obtenidas de manera deductiva (general a lo particular).

\section{Reflexiones finales}

La pretensión de este análisis, es apenas intentar reflexionar acerca de la frecuente reorganización del conocimiento, con tendencias marcadas hacia mayores cotas de especialización disciplinar -sin descartar la superespecialización a partir de la división y subdivisión de áreas tradicionales del conocimiento-, como consecuencia lógica del trabajo y la profundización de la investigación científica, reconociendo y valorando el papel fundamental que cumple en la explicación de un sinfín de complejos problemas, producto de la revolución científica experimentada con mayor notoriedad desde fines del siglo XX e inicios del siglo XXI.

Los fenómenos de la superespecialización y los procesos de interdisciplinariedad del conocimiento, no sólo han contribuido a la conformación de equipos de investigación, sino fundamentalmente a la adopción de nuevos y

\footnotetext{
${ }^{7}$ Adoptamos el término apertura como aquella posibilidad verosímil de "abrir", de lograr dejar al descubierto aquello que se encuentra oculto, con la única intención de otorgarle cierta autenticidad y originalidad.
} 
complejos modelos de análisis mucho más potentes que hacen inviable seguir recurriendo a los modelos tradicionales de una única especialización disciplinar, debido en gran manera, al reconocimiento de la complejidad del mundo y la cultura, en una clara intencionalidad de evitar que los resultados se vean afectados por reduccionismos, fragmentaciones y hasta deformaciones en su abordaje y análisis y, por otro, incrementar las capacidades de descripción teórica y empírica de todo aquello que sucede en el objeto de análisis.

En esta dinámica, la función investigativa se ha visto ampliamente favorecida a consecuencia de la instalación definitiva de la ciencia y el conocimiento como componentes estructurales que cualifican el desarrollo de las fuerzas productivas de las sociedades. Situación privilegiada que ha dado lugar a considerarla como la herramienta esencial para desarrollar el conocimiento, por ser el mecanismo privilegiado que posibilita la comunicación y la transferencia tecnológica a diferentes campos disciplinares, contextos y regiones del mundo, cuya complejidad es ampliamente visualizada.

El reconocimiento de la complejidad del mundo y la cultura, exige a los hombres de ciencia y en general a la comunidad científica, desentrañar los problemas desde múltiples ópticas de abordaje, la adopción de nuevos y complejos modelos de análisis tendientes a incrementar las capacidades de descripción teórica y empírica de todo aquello que sucede en la realidad del mundo social desde miradas inter y transdisciplinares.

De igual modo, se intenta reflexionar acerca de la constitución de la ciencia de institución o saber científico, que gracias a la epistemología contemporánea ha permitido superar el prejuicio positivista que planteaba que la ciencia era el único saber. Hoy puede afirmarse que sólo representa una forma más de conocimiento, dado que existen muchos otros saberes (conocimientos) que no son científicos (saberes populares), pero que tampoco pretenden serlo.

Concebir la ciencia bajo estas nuevas miradas, exige en primer término descolonizar el poder del saber y del ser impuestos por el imperialismo cultural hegemónico, que ha socavado $y$ penetrado intensamente en nuestras mentalidades, pensamientos y subjetividades, quebrantando con ello, la profunda y rica densidad histórica y cultural que poseen los pueblos; la marginalización de los grupos sociales que deliberadamente han sido puestos en segundo plano de interés para preservar el statu quo de ese imperialismo científico hegemónico imperante.

En segundo término, es preciso construir puentes de encuentros dialógicos horizontales entre saberes prácticos considerados nocientíficos y aquellos considerados científicos; de tal forma que ambas puedan enriquecerse recíprocamente y producto de ese encuentro, sea viable la conformación de comunidades epistémicas cuyo ideal supremo no sea otro que el de ir construyendo al desarrollo de ciencia y conocimiento útil y contextualizado.

Con ello, se pretende asumir algunos reparos de orden no sólo conceptual sino principalmente de orden epistemológico, en tanto que el conocimiento se desarrolla en un contexto sociohistórico determinado, pero que también se halla cargada de orientaciones de orden ideológico y valorativo que la condicionan y que van más allá del mero planteamiento que ella representa como posibilidad viable y válida de producción y construcción de conocimiento, resultante de la negación y superación progresiva de incertidumbres; así como la transferencia de conocimiento que hace referencia a un proceso articulado, desde el conocimiento existente hacia el que se produce y recrea en su utilidad pública y social.

De esta forma, resulta acertado plantear que, en todo análisis crítico-reflexivo sobre la constitución de las Ciencias, debe considerarse su origen en lo social; pues no debe olvidarse que son construcciones genuinamente sociales, resultantes del hacer y quehacer social (hechos sociales), ligados a determinados condicionamientos de poder bajo los cuales se producen, difunden y aplican.

Por lo tanto, el saber científico es un saber cargado de ideología, de intencionalidades u orientaciones valorativas (intereses, motivaciones e inclinaciones), propias de los investigadores en tanto sujetos sociales, que formulan explicaciones que intentan pensar la realidad social mediante la definición y 
construcción de un corpus teórico e instrumentos, producto del trabajo científico en la pretensión de acercarse a la comprensión e interpretación de la realidad, aunque ésta nunca sea alcanzada en su totalidad.

Por ello, urge desarrollar en el pensamiento científico, una conciencia crítica-reflexiva de reconocimiento, en el sentido de hallarnos cada vez más cerca de una incomprensión de la totalidad, de la completitud; que jamás llegaremos a poseer una comprensión cabal de la misma y que nos encontramos cada vez más cerca de parcialidades de una verdad que es provisoria y no así definitoria. 


\section{Referencias}

Aisenberg, B.; Alderoqui, S. (Comps.). (2007). Didáctica de las Ciencias Sociales. Buenos Aires: Paidós.

Bourdieu, J. P. (1997). Razones Prácticas Sobre la Teoría de la Acción. Barcelona: Anagrama.

- (2003). El Oficio del Científico. Ciencia de la Ciencia y Reflexividad. Barcelona: Anagrama.

De Sousa Santos, B. (2008). Pensar el Estado y la Sociedad: Desafíos Actuales. La Paz: Clacso-Muela del diablo editores.

- (2007). La Universidad en el Siglo XXI. La Paz: Cides-UMSA-ASDI- Plural editores.

Di Tella, T.; Chumbita, H.; Gamba, S.; Gajardo, P. (2008). Diccionario de Ciencias Sociales y Políticas. Buenos Aires: Emece.

Follari, R. (2000). Epistemología y Sociedad. Santa Fe: Homo Sapiens.

Mattelart, A. (2006). Diversidad Cultural y Mundialización. Barcelona: Paidós ibérica.

Milner, J. C. (1996). La Obra Clara. Lacan, la Ciencia, la Filosofía. Buenos Aires: Bordes manantial.

Morin, E. (1995). Sociología. Madrid: Tecnos.

Pourtois, J. P.; Desmet, H. (1992). Epistemología e Instrumentación en Ciencias Humanas. Barcelona: Herder.

Saavedra, J. L. (2011). Descolonización y Decolonialidad de la Universidad. En M. Navarro, Estrategias Para una Educación Superior Descolonizadora Intra e Intercultural. (Seminario-Taller Cbba, 1818/11/2010). Cochabamba: FUNPROEIB ANDES.

Scribano, A. (2009). Estudios Sobre Teoría Social Contemporánea. Buenos Aires: Ciccus. 